\title{
Liver Cirrhosis Mortality Worldwide: in Hospital Factors Associated?
}

\author{
Chyntia Olivia Maurine Jasirwan \\ Division of Hepatobiliary, Department of Internal Medicine, Faculty of Medicine \\ Universitas Indonesia/Dr. Cipto Mangunkusumo General National Hospital, Jakarta
}

Liver cirrhosis has become one of the leading causes of morbidity and mortality. It killed over a million people worldwide in 2010, according to the Global Burden of Disease (GBD), up from 676,000 deaths in 1980. It has a low survival rate, so data on the incidence of geographical variations is critical for preventing its associated disability and mortality. However, because relevant data for 58/187 (31\%) of countries were not available in the report, this medical condition's heavy burden maybe even more significant. ${ }^{1}$

Liver cirrhosis is the most common cause of liver-related death in the world. ${ }^{2}$ It is the final stage of progressive liver fibrosis, in which the hepatic architecture has become distorted. ${ }^{3}$ Cirrhosis is compensated in the early stages. At this stage, most patients are asymptomatic, and cirrhosis is typically discovered incidentally during medical encounters for other reasons. As a result, reports on the prevalence of compensated cirrhosis are almost always understated. Decompensation in patients with compensated cirrhosis is typically defined as the first occurrence of ascites, oesophageal variceal bleeding, hepatic encephalopathy, and, in some cases, liver failure. ${ }^{4,5}$

These patients are quickly referred to the physician due to the nature of decompensation so that reports of decompensated cirrhosis are probably significantly more accurate than those of compensated cirrhosis. ${ }^{4}$ Once decompensation occurs, mortality and morbidity associated with the increase of cirrhosis and the 1-year case-life rate may reach up to 80 percent depending on the cause of decompensation. ${ }^{6,7}$ The decompensated type is responsible for almost all cirrhosis-related mortality and morbidity. Such patients require frequent healthcare and a growing number of medicines over time.

The repeated hospitalizations harm one's quality of life (admissions and stays). ${ }^{8}$ As the disease progresses, hospital stays become more frequent and more prolonged. Finally, patients either die or undergo a liver transplant, which is an expensive option for patients, healthcare services, health funding, and health governance. $^{8}$

Tapper EB et al performed a cohort study that showed comorbidity patterns of death in patients who died from cirrhosis. Conversely, while no statistical increment was seen in the numbers of comorbid cirrhosis and hepatorenal syndrome deaths $(95 \% \mathrm{CI}$ : $0.2-3.7 \%$ ), a slight (but statistically significant) rise was seen in the cirrhosis with sepsis and peritonitis $(2.5 \%$ to $4.0 \%$ ). About $60 \%$ of the 472,290 people in that age group had sepsis or peritonitis. Regardless of age, the mortality elevated, but the increase was not significant. Those aged 25 to 44 experienced a substantial raises in sepsis or peritonitis-related mortality, which started in 2009 , had annual percentage changes of $13.5 \%$ (from $9.4 \%$ to $17.4 \%$ ) and (4.3\% to $10.6 \%$ ). Also, people who were 75 or older at the end of 2013 had a $3.3 \%$ higher mortality due to sepsis and peritonitis between $2.0 \%$ and $4.6 \%{ }^{9}$

Studies performed in 2 different tertiary hospitals in Jakarta, Indonesia, showed the same pattern. Gani et al showed the study included all 89 patients, and among them, $78.3 \%$ were died because of infection after two years of follow-up. This risk factor was rather different from other studies conducted by Darnindro et al, which showed that among 41 liver cirrhosis patients, the average age was 52.9, and the percentage of patients who died during hospitalization was $12.2 \%$. Patients were admitted to the hospital due to various complaints, and the most common complaint was gastrointestinal bleeding. Creatinine level $>1.3 \mathrm{mg} / \mathrm{dL}$ had a higher mortality risk than creatinine below $1.2 \mathrm{mg} / \mathrm{dL} .^{10,11}$

Roberts and colleagues based their results on 8,192 patients admitted to hospitals in the Oxford area of Southern England. The results were focused on discharge and death certificate statistics. The mortality rate was $34 \%$ one year after admission and stayed that way for the entire 30 -year observation period. One year after admission, the standardized mortality rate was 16.3 times that of the general population. These 
results were immune to multiple analytical techniques. Over the 30 years, both the overall community of 8,192 patient admissions and the diagnostic subgroups individually produced disappointing results. The data demonstrate how lethal liver cirrhosis has been and continues to be. ${ }^{12}$

Why does cirrhosis mortality remain constant, despite our belief that it is decreasing? We foresee lower mortality as a result of the introduction of various new therapies. During the study era, interventions such as liver transplantation, endoscopic treatment for bleeding varices, prevention of bleeding and rebleeding from varices with beta-blockers, antiviral drugs for hepatitis $\mathrm{B}$ or $\mathrm{C}$, and antibiotics for preventing and treating cirrhosis complications were all implemented.

As often, it could be wrong when things do not add up. Might this be a mixture of misconduct, false expectations, and misapplication? Further epidemiological studies in the UK and abroad are needed compared to other countries and the results of Roberts and colleagues and the increased rates of liver cirrhosis mortality in England over time. This research may also analyze improvements in mortality over five and ten years, in addition to a one-year mortality rate.

Our standards may also have to be adapted to the intervention results. It might not be as effective as we imagine to intervene. Therefore, more successful measures must be developed as a matter of urgency. Besides seeking new interventions for any liver cirrhosis, lifestyle intervention in non-alcoholic liver disease should be strengthened. We also need to analyze whether the intervention we use in our clinical practice has beneficial and adverse effects. We need to collaborate even more to achieve these advancements. We can perform much larger randomized clinical trials with a far improved methodology by collaboration.

In conclusion, however, mortality rates varied across countries, owing primarily to the prevalence of risk factors for the disease, such as the amount and pattern of alcohol consumption, control of metabolic syndrome, and viral hepatitis. Cirrhosis decompensation can manifest acutely as ascites, hepatic encephalopathy, gastrointestinal hemorrhage, acute renal failure, and bacterial infections that necessitate hospitalization and further aggravate mortality.

\section{REFERENCES}

1. Mokdad AA, Lopez AD, Shahraz S, Lozano R, Mokdad AH, Stanaway J. Liver cirrhosis mortality in 187 countries between 1980 and 2010: a systematic analysis. BMC Medicine 2014;12:

2. Roth GA, Abate D, Abate KH, et al. Global, regional, and national age-sex-specific mortality for 282 causes of death in 195 countries and territories, 1980-2017: a systematic analysis for the Global Burden of Disease Study 2017. Lancet 2018;392:1736-88.

3. Anthony PP, Ishak KG, Nayak NC, Poulsen HE, Scheuer PJ, Sobin LH. The morphology of cirrhosis. Recommendations on definition, nomenclature, and classification by a working group sponsored by the World Health Organization. J Clin Pathol 1978;31:395-414.

4. Fleming KM, Aithal GP, Card TR, West J. The rate of decompensation and clinical progression of disease in people with cirrhosis: a cohort study. Aliment Pharmacol Ther 2010;32:1343-50.

5. Moreau R, Jalan R, Gines P, Pavesi M, Angeli P, Cordoba J, et al. Acute-on-chronic liver failure is a distinct syndrome that develops in patients with acute decompensation of cirrhosis. Gastroenterology 2013;144:1426-37.e1-9.

6. D'Amico G, Garcia-Tsao G, Pagliaro L. Natural history and prognostic indicators of survival in cirrhosis: a systematic review of 118 studies. J Hepatol 2006;44:217-31.

7. Fleming KM, Aithal GP, Card TR, West J. All-cause mortality in people with cirrhosis compared with the general population: a population-based cohort study. Liver Int 2012;32:79-84.

8. Ge PS, Runyon BA. Treatment of patients with cirrhosis. N Engl J Med 2016;375:767-77.

9. Tapper EB, Parikh ND. Mortality due to cirrhosis and liver cancer in the United States, 1999-2016: an observational study. BMJ 2018;362:k2817.

10. Gani RA. Survival analysis of hospitalized liver cirrhotic patients in Jakarta: 2 years follow up study. Indones $\mathrm{J}$ Gastroenterol Hepatol Dig Endosc 2021;22:9-15.

11. Darnindro N, Manurung A, Mulyana E, Harahap A. Clinical characteristics of liver cirrhosis patients in internal medicine inpatient ward of fatmawati general hospital and factors affecting mortality during hospitalization. Indones $\mathrm{J}$ Gastroenterol Hepatol Dig Endosc 2021;22:3-8.

12. Roberts SE. Trends in mortality after hospital admission for liver cirrhosis in an English population from 1968 to 1999. Gut 2005;54:1615-21. 